\title{
On the behavior of lightning current and charge density in the case of tall tower
}

\begin{abstract}
Considering on the behavior of charge density in the case of strikes of lightning to tall tower is an important issue and it can be applied in the electrical protection of equipment (on the tower) and also lightning electromagnetic fields due to tower and channel. In this paper, the behaviors of charge density under the different tower and channel conditions were considered and the results were discussed accordingly. The results showed that the tower height and tower reflection factor are strongly effective on the values of charge density due to additional reflected currents along towering a channel, however other parameters of the tower and channel can be effective on the values of charge density that were studied in this paper.
\end{abstract}

Keyword: Lightning; Return stroke current; Charge density 